\title{
On the classification of rational four-dimensional unital division algebras
}

\author{
Gustav Hammarhjelm
}

August 16, 2021

\begin{abstract}
In 4], the category $\mathscr{C}(k)$ of four-dimensional unital division algebras, whose right nucleus is non-trivial and whose automorphism group contains Klein's four group $V$, is studied over a general ground field $k$ with char $k \neq 2$. In particular, the objects in $\mathscr{C}(k)$ are exhaustively constructed from parameters in $k^{3}$ and explicit isomorphism conditions for the constructed objects are found in terms of these parameters.

In this paper, we specialize to the case $k=\mathbb{Q}$ and present results towards a classification of $\mathscr{C}(\mathbb{Q})$. In particular, for each field $\ell$ with $[\ell: k]=2$ we present explicity a two-parameter family of pairwise non-isomorphic non-associative objects in $\mathscr{C}(\mathbb{Q})$ that admit $\ell$ as a subfield and we provide a method for classifying the full subcategory of central skew fields admitting $\ell$ as a subfield and $k V$-submodule. We also classify the subcategory of $\mathscr{C}(\mathbb{Q})$ of all fourdimensional Galois extensions of $\mathbb{Q}$ with Galois group $V$ that admit $\ell$ as a subfield.
\end{abstract}

\section{Background and setup}

In this paper, an algebra over a field $k$, or more briefly, a $k$-algebra, is a $k$-vector space $A$ equipped with a bilinear multiplication map, written by juxtaposition as $(a, b) \mapsto a b$ for $a, b \in A$. Note that we do not require $A$ to be associative. A $k$ algebra $A$ is said to be a division algebra if $A \neq\{0\}$ and the linear endomorphisms $x \mapsto a x$ and $x \mapsto x a$ of $A$ are bijective for all $a \in A \backslash\{0\}$.

Given a category $\mathscr{C}$ such that the collection of isoclasses of $\mathscr{C}$ forms a set, an interesting problem is to classify $\mathscr{C}$. In this paper, a classification of $\mathscr{C}$ is understood to be an explicitly given transversal of the isoclasses of $\mathscr{C}$, that is, an explicitly given exhaustive and irredundant set of representatives. A challenging problem, open in most cases, is trying to classify categories of division algebras; for classifications of particular categories of division algebras, see e.g. [1], [2], [3].

Let $k$ be a field and let $V$ be Klein's four group. Let $\mathscr{C}(k)$ be the category of four-dimensional unital division $k$-algebras $A$ such that $V \subset \operatorname{Aut}_{k}(A)$ and

$$
N_{r}(A):=\{n \in A \mid(a b) n=a(b n), \forall a, b \in A\},
$$


the right nucleus of $A$, is non-trivial, meaning $k \subsetneq N$. The morphisms in $\mathscr{C}(k)$ are the non-zero algebra morphisms. These are injective due to the division property, hence bijective, as they are endomorphisms of a finite-dimensional vector space. It follows that $\mathscr{C}(k)$ is a groupoid, i.e. a category in which all morphisms are isomorphisms. Note that $V \subset \operatorname{Aut}_{k}(A)$ gives $A$ the structure of a $k V$-module.

For a given field $k$, let $\mathscr{Q}(k)$ denote the category of all field extensions $k \subset \ell$ with $[\ell: k]=2$, in which morphisms are the $k$-linear field morphisms. Hereafter a field $k$ with char $k \neq 2$ and $\ell \in \mathscr{Q}(k)$ are fixed. Let $\bar{x}$ denote the Galois conjugate of $x \in \ell$ and let $n_{\ell / k}(x)=x \bar{x}$ be the norm of the field extension $k \subset \ell$.

For $c:=\left(c_{1}, c_{2}, c_{3}\right) \in k^{3}$ and $x, y \in \ell$ let

$$
M_{c}(x, y)=\left(\begin{array}{cc}
x & c_{2} y+c_{3} \bar{y} \\
y & \left(1-c_{1}\right) x+c_{1} \bar{x}
\end{array}\right) \in \ell^{2 \times 2}
$$

and construct the four-dimensional $k$-algebra $A(\ell, c)$ by $A(\ell, c)=\ell^{2}$ as a vector space over $k$ and multiplication given by

$$
\left(\begin{array}{l}
x \\
y
\end{array}\right)\left(\begin{array}{c}
z \\
w
\end{array}\right)=M_{c}(x, y)\left(\begin{array}{c}
z \\
w
\end{array}\right)
$$

for $x, y, z, w \in \ell$, where the right hand side of (2) is ordinary multiplication of $\ell$-matrices. For the remainder of this paper, every occurrence of the word "construction" refers to the above construction of $A(\ell, c)$. Define $C_{\ell}=\left\{c \in k^{3} \mid\right.$ $A(\ell, c)$ is a division algebra $\}$ and call $C_{\ell}$ the set of admissible triples. Equivalently, $c$ is admissible if and only if $\operatorname{det} M_{\ell, c}(x, y) \neq 0$ for all $(x, y) \in \ell^{2} \backslash\{(0,0)\}$.

For $\gamma \in \operatorname{Aut}_{k}(A)$ and $\lambda \in k$, let $E_{\lambda}(\gamma)=\{x \in A \mid \gamma(x)=\lambda x\}$. As in [4], let

$\mathscr{C}_{\ell}(k)=\left\{A \in \mathscr{C}(k) \mid \exists n \subset A, \exists \alpha, \beta \in \operatorname{Aut}_{k}(A): \ell \cong n=E_{1}(\beta) \subset N_{r}(A),\langle\alpha, \beta\rangle \cong V\right\}$.

From [4, Prop. 4.1] it is seen that

$$
\mathscr{C}_{\ell}(k)=\{A \in \mathscr{C}(k) \mid \exists n \subset A, n \cong \ell \text { and } n \text { is a } k V \text {-submodule of } A\} .
$$

By [4, Theorem 4.5] each $A \in \mathscr{C}_{\ell}(k)$ is isomorphic to $A(\ell, c)$ for some $c \in C_{\ell}$.

Due to [4, Prop. 2.2] the category $\mathscr{C}(k)$ decomposes as a coproduct $\mathscr{C}(k)=$ $\mathscr{K}(k) \amalg \mathscr{S}(k) \amalg \mathscr{N}(k)$ of the full subcategories $\mathscr{K}(k), \mathscr{S}(k), \mathscr{N}(k)$ consisting of field extensions, skew fields that are central over $k$ and algebras with right nucleus of dimension 2, respectively. Note that the algebras in $\mathscr{N}(k)$ are not associative. If $\mathscr{L}$ is a classifying list of $\mathscr{Q}(k)$ we have, from [4, Prop 4.8], that

$$
\mathscr{C}(k)=\left(\bigcup_{\ell \in \mathscr{L}} \mathscr{K}_{\ell}(k)\right) \amalg\left(\bigcup_{\ell \in \mathscr{L}} \mathscr{S}_{\ell}(k)\right) \amalg\left(\coprod_{\ell \in \mathscr{L}} \mathscr{N}_{\ell}(k)\right)
$$

where $\mathscr{K}_{\ell}(k), \mathscr{S}_{\ell}(k), \mathscr{N}_{\ell}(k)$ will be called local subcategories and are defined as intersections of $\mathscr{C}_{\ell}(k)$ with the corresponding categories $\mathscr{K}(k), \mathscr{S}(k), \mathscr{N}(k)$.

Given a ring $R$ and $S \subset R$ set $S^{*}=S \backslash\{0\}$ and $S_{\mathrm{sq}}=\left\{s^{2} \mid s \in S\right\}$. Given a group $G$ with identity $e$ we set $G^{\circ}=G \backslash\{e\}$ and call $G^{\circ}$ a punctured group. Combining [4, Lemma 3.4, Corollary. 5.4] we reproduce the following result. 
Proposition 1.1. Let $k$ be a field with char $k \neq 2, \ell \in \mathscr{Q}(k)$ and $c, d \in C_{\ell}$.

(i) We have $A(\ell, c) \cong A(\ell, d)$ in $\mathscr{C}(k)$ if and only if there exists $x \in \ell^{*}$ such that $\left(c_{1}, c_{2}, c_{3}\right)=\left(d_{1}, x^{2} d_{2}, n_{\ell / k}(x) d_{3}\right)$.

(ii) If $S(\ell) \subset k^{*}$ is a transversal of $\left(k^{*} /\left(k^{*} \cap \ell_{\mathrm{sq}}\right)\right)^{\circ}$ then $\{A(\ell,(0, s, 0)) \mid s \in S(\ell)\}$ classifies $\mathscr{K}_{\ell}(k)$.

(iii) If $T(\ell) \subset k^{*}$ is a transversal of $\left(k^{*} / n_{\ell / k}\left(\ell^{*}\right)\right)^{\circ}$ then $\{A(\ell,(1,0, t)) \mid t \in T(\ell)\}$ classifies $\mathscr{S}_{\ell}(k)$.

Part (i) of Proposition 1.1 is an isomorphism condition of algebras constructed from admissible triples. Parts (ii), (iii) of the proposition provide reductions of the local classification problems in $\mathscr{C}(k)$ concerning fields and skew fields to finding transversals of punctured quotient groups of $k$.

In [4, Section 5] the category $\mathscr{C}(k)$ is classified in the case of $k$ being a finite field of odd order or $k$ an ordered field with $k_{\mathrm{sq}}=k_{\geq 0}$. In this paper the case $k=\mathbb{Q}$ is discussed.

\section{On the classification of $\mathscr{C}(\mathbb{Q})$}

In this section we investigate the category $\mathscr{C}(\mathbb{Q})$ by studying the local parts of the covering (3). In Section 2.1 the object of study is $\mathscr{K}(\mathbb{Q})$ and in Proposition 2.1 we classify $\mathscr{K}(\ell / \mathbb{Q})$ for each $\ell$ in a classifying list $\mathscr{L}$ (to be specified below) of $\mathscr{Q}(\mathbb{Q})$. The classifications are found by explicitly presenting transversals as in Proposition 1.1 (ii).

In Section 2.2 we study $\mathscr{S}(\mathbb{Q})$ and we construct an exhaustive list of $\left(\mathbb{Q}^{*} / n_{\ell / \mathbb{Q}}\left(\mathbb{Q}^{*}\right)\right)^{\circ}$ for each $\ell \in \mathscr{L}$. We provide a method for reducing the exhaustive list to a transversal and we hence approach a classification of $\mathscr{S}_{\ell}(\mathbb{Q})$ in view of Proposition 1.1 (iii). In Proposition 2.3 we classify $\mathscr{S}_{\ell}(\mathbb{Q})$ in the special case $\ell=\mathbb{Q}(i)$. The results in this section rely on classic number theoretic theorems dating back to Fermat and Legendre.

In Section 2.3 we study $\mathscr{N}(\mathbb{Q})$ and present for each $\ell \in \mathscr{L}$ an irredundant twoparameter family $\tilde{P}(\ell) \subset C_{\ell}$ of admissible triples such that $A(\ell, c) \in \mathscr{N}_{\ell}(k)$ for each $c \in \tilde{P}(\ell)$. In the special case $\ell=\mathbb{Q}(i)$ we enlarge the two-parameter family to an irredundant four-parameter family.

Notation: Besides the notation introduced just before Proposition [1.1 we also set $\mathbb{N}=\mathbb{Z}_{\geq 0}$ and define $N_{2}=\mathbb{N}_{\text {sq }}+\mathbb{N}_{\text {sq }}, Q_{2}=\mathbb{Q}_{\text {sq }}+\mathbb{Q}_{\text {sq }}$, i.e. the natural and rational numbers expressible as a sum of two squares, respectively. Let $\mathbb{P} \subset \mathbb{N}$ denote the set of prime numbers and let $\mathbb{P}_{1}, \mathbb{P}_{3}$ denote the subsets of prime numbers $\equiv 1,3(\bmod 4)$ respectively. Let $m_{p}(n)=\max \left\{k \in \mathbb{N}: p^{k} \mid n\right\}$ for $p \in \mathbb{P}, n \in \mathbb{Z} \backslash\{0\}$. In addition, we adopt the convention that $m_{p}(0)=\infty$ for every $p \in \mathbb{P}$.

Let $Z=\left\{n \in \mathbb{Z} \mid m_{p}(n) \leq 1\right.$ for all $\left.p \in \mathbb{P}\right\} \backslash\{1\}$ so that $Z$ is the set of all square-free integers except 1 . Then, $Z$ is a transversal of $\left(\mathbb{Q}^{*} / \mathbb{Q}_{\mathrm{sq}}^{*}\right)^{\circ}$ and the list $\mathscr{L}:=\{\mathbb{Q}(\sqrt{z}) \mid z \in Z\}$ classifies $\mathscr{Q}(\mathbb{Q})$. 


\subsection{On $\mathscr{K}(\mathbb{Q})$}

For each $\ell \in \mathscr{L}$, transversals $S(\ell)$ as in Proposition 1.1 (ii) are given as follows.

Proposition 2.1. Take $z \in Z$ and set $\ell=\mathbb{Q}(\sqrt{z}) \in \mathscr{L}$.

(i) If $z=-1$, then $S(\ell)=Z_{<0} \backslash\{-1\}$ is a transversal of $\left(\mathbb{Q}^{*} /\left(\mathbb{Q}^{*} \cap \ell_{\mathrm{sq}}\right)\right)^{\circ}$.

(ii) If $z \neq-1$, then $S(\ell)=\{w \in Z:|\operatorname{gcd}(z, w)|<\sqrt{|z|}\}$ is a transversal of $\left(\mathbb{Q}^{*} /\left(\mathbb{Q}^{*} \cap \ell_{\mathrm{sq}}\right)\right)^{\circ}$.

Proof. Let $\pi: \mathbb{Q}^{*} \longrightarrow\left(\mathbb{Q}^{*} /\left(\mathbb{Q}^{*} \cap \ell_{\mathrm{sq}}\right)\right)^{\circ}$ denote the quotient map. We have $\mathbb{Q}^{*} \cap \ell_{\mathrm{sq}}=$ $\mathbb{Q}_{\mathrm{sq}}^{*} \cup z \mathbb{Q}_{\mathrm{sq}}^{*}$.

(i): Take $q \in \mathbb{Q}^{*}$ with $\pi(q) \neq \pi(z)$ and $w \in Z \backslash\{z\}$ such that $\pi(q)=\pi(w)$. We have $\pi(q)=\pi( \pm w)$ where either $w \in S(\ell)$ or $-w \in S(\ell)$, so $S(\ell)$ exhausts $\left(\mathbb{Q}^{*} /\left(\mathbb{Q}^{*} \cap \ell_{\mathrm{sq}}\right)\right)^{\circ}$. Suppose that $w, w^{\prime} \in S(\ell)$ and $\pi(w)=\pi\left(w^{\prime}\right)$. Then, as $w, w^{\prime}$ have the same sign we have $\frac{w}{w^{\prime}} \in \mathbb{Q}_{\mathrm{sq}}^{*}$ so there are integers $r, s$ with $\operatorname{gcd}(r, s)=1$ such that $s^{2} w=r^{2} w^{\prime}$. Take $p \in \mathbb{P}$ such that $p \mid w$. Then $p \mid r^{2} w^{\prime}$ and if $p \mid r$ we get $p^{2} \mid s^{2} w$ and since $w$ is square-free we get $p \mid s$, contradiction to $\operatorname{gcd}(r, s)=1$. Thus $p \mid w^{\prime}$ and by symmetry we get $p \mid w$ if $p \mid w^{\prime}$, hence $w=w^{\prime}$. Thus $S(\ell)$ is a transversal of $\left(\mathbb{Q}^{*} /\left(\mathbb{Q}^{*} \cap \ell_{\mathrm{sq}}\right)\right)^{\circ}$.

(ii): Suppose now $z \neq-1$ and take $q \in \mathbb{Q}^{*}$ with $\pi(q) \neq \pi(z)$. As above, find $w \in Z \backslash\{z\}$ with $\pi(q)=\pi(w)$ and write $w=d w^{\prime}$ with $d=\operatorname{gcd}(z, w)>0$. If $d<\sqrt{|z|}$ then $w \in S(\ell)$. Otherwise, $\left|\frac{z}{d}\right|<\sqrt{|z|}$. Indeed, if $d \geq \sqrt{|z|}$ and $\left|\frac{z}{d}\right| \geq \sqrt{|z|}$ then we must have $d=\left|\frac{z}{d}\right|=\sqrt{|z|}$ so that $\sqrt{|z|}$ is an integer, contradiction to $z$ being a square-free integer different from \pm 1 . Then, $\frac{z}{d} w^{\prime} \in S(\ell)$ and $\pi\left(\frac{z}{d} w^{\prime}\right)=$ $d^{2} \frac{z}{d} w^{\prime}\left(\mathbb{Q}_{\mathrm{sq}}^{*} \cup z \mathbb{Q}_{\mathrm{sq}}^{*}\right)=w\left(\mathbb{Q}_{\mathrm{sq}}^{*} \cup z \mathbb{Q}_{\mathrm{sq}}^{*}\right)=\pi(q)$. Hence, $S(\ell)$ exhausts $\left(\mathbb{Q}^{*} /\left(\mathbb{Q}^{*} \cap \ell_{\mathrm{sq}}\right)\right)^{\circ}$.

To prove irredundance of $S(\ell)$, take $w_{1}, w_{2} \in S(\ell)$ and suppose that $\pi\left(w_{1}\right)=$ $\pi\left(w_{2}\right)$. Then, either $\frac{w_{1}}{w_{2}} \in \mathbb{Q}_{\mathrm{sq}}^{*}$ or $\frac{w_{1}}{w_{2}} \in z \mathbb{Q}_{\mathrm{sq}}^{*}$. For $i \in\{1,2\}$, write $w_{i}=d_{i} w_{i}^{\prime}$ with $d_{i}=\operatorname{gcd}\left(w_{i}, z\right)>0$ and $\left|d_{i}\right|<\sqrt{|z|}$.

Suppose first that $\frac{w_{1}}{w_{2}} \in \mathbb{Q}_{\mathrm{sq}}^{*}$. Then, there are integers $r, s$ with $\operatorname{gcd}(r, s)=1$ such that $w_{1}=\frac{r^{2}}{s^{2}} w_{2}$ which implies $s^{2} d_{1} w_{1}^{\prime}=r^{2} d_{2} w_{2}^{\prime}$. Suppose there is $p \in \mathbb{P}$ with $p \mid d_{1}$. Since $d_{1} \mid z$ and $\operatorname{gcd}\left(z, w_{2}^{\prime}\right)=1$ we have $p \mid r^{2} d_{2}$. If $p \mid r$ then $p^{2} \mid r^{2}$ and so $p^{2} \mid s^{2} d_{1} w_{1}^{\prime}=s^{2} w_{1}$, contradiction to $w_{1}$ being square-free. Hence, $p \mid d_{2}$. By symmetry, $p \mid d_{2}$ implies $p \mid d_{1}$ and hence $d_{1}=d_{2}$. Thus, $s^{2} w_{1}^{\prime}=r^{2} w_{2}^{\prime}$ and by a similar argument, we get $w_{1}^{\prime}=w_{2}^{\prime}$ and hence also $w_{1}=w_{2}$ as desired.

Suppose now $\frac{w_{1}}{w_{2}} \in z \mathbb{Q}_{\mathrm{sq}}^{*}$ so that we can write $s^{2} d_{1} w_{1}^{\prime}=r^{2} z d_{2} w_{2}^{\prime}$ for some relatively prime integers $r, s$. Using $\operatorname{gcd}\left(z, w_{i}^{\prime}\right)=1$ and arguments as above, we get $w_{1}^{\prime}= \pm w_{2}^{\prime}$ and hence $s^{2} d_{1}= \pm r^{2} z d_{2}$. Thus, $\frac{r^{2}}{s^{2}}= \pm \frac{z}{d_{1}} \cdot d_{2} \in \mathbb{N}$. Hence, $\pm \frac{z}{d_{1}} \cdot d_{2}$ is the square of an integer, and as $\frac{z}{d_{1}}, d_{2}$ are both square-free we must have $\pm \frac{z}{d_{1}}=d_{2}$ so that $|z|=d_{1} d_{2}$, which is a contradiction since $d_{1}, d_{2}<\sqrt{|z|}$ by assumption.

Remark. By Proposition 1.1 (ii) we arrive, for each $\ell \in \mathscr{L}$, at a classification of the category $\mathscr{K}_{\ell}(\mathbb{Q})$, which consists of all 4 -dimensional Galois extensions of $\mathbb{Q}$ with Galois group $V$ that contain $\ell$ as a subfield. 


\subsection{On $\mathscr{S}(\mathbb{Q})$}

Given a field $\ell=\mathbb{Q}(\sqrt{z}) \in \mathscr{L}$ we attempt to find a transversal $T(\ell)$ as in Proposition 1.1 (iii). The set $Z \backslash n_{\ell / \mathbb{Q}}\left(\ell^{*}\right)$ exhausts $\left(\mathbb{Q}^{*} / n_{\ell / \mathbb{Q}}\left(\ell^{*}\right)\right)^{\circ}$ and in order to present this set explicitly we have the following result.

Proposition 2.2. Take $z \in Z$ and set $\ell=\mathbb{Q}(\sqrt{z})$. Take $w \in Z$ and set $0<d=$ $\operatorname{gcd}(z, w)$. Then, $w \in n_{\ell / \mathbb{Q}}\left(\ell^{*}\right)$ if and only if all of the following four conditions are satisfied: $z, w$ are not both negative, $z$ is a square $\bmod \frac{w}{d}, w$ is a square mod $\frac{z}{d}$ and $-\frac{z w}{d^{2}}$ is a square mod $d$.

Proof. We have that $w \in n_{\ell / \mathbb{Q}}\left(\ell^{*}\right)$ if and only if there exists $(a, b) \in \mathbb{Q}^{2} \backslash\{(0,0)\}$ such that $w=a^{2}-z b^{2}$. We claim that the following are equivalent:

(i) There exists $(a, b) \in \mathbb{Q}^{2} \backslash\{(0,0)\}$ such that $w=a^{2}-z b^{2}$.

(ii) There exists $(a, b, c) \in \mathbb{Z}^{3} \backslash\{(0,0,0)\}$ such that $a^{2}-w b^{2}-z c^{2}=0$.

(iii) There exists $(a, b, c) \in \mathbb{Z}^{3} \backslash\{(0,0,0)\}$ such that $d a^{2}-\frac{w}{d} b^{2}-\frac{z}{d} c^{2}=0$.

Indeed, suppose $(a, b)$ satisfies (i). If $a=0$ then $\frac{z}{w} \in \mathbb{Q}_{\text {sq }}$ then it is immediate that (ii) holds. Otherwise, write $b=\frac{b_{1}}{b_{2}}, a=\frac{a_{1}}{a_{2}}, a_{1} \neq 0$. Then $a_{1}^{2}-z b_{1}^{2}-w\left(a_{2} b_{2}\right)^{2}=0$, so (ii) holds. Conversely, if $(a, b, c)$ satisfies (ii), then $b \neq 0$, for otherwise $c \neq 0$ and $z=\left(\frac{a}{c}\right)^{2}$, contradicting $z$ square-free. With $b \neq 0$ we see that $w=\left(\frac{a}{b}\right)^{2}-z\left(\frac{c}{b}\right)^{2}$, so (i) holds.

Suppose $(a, b, c)$ satisfies (ii). Since $d \mid z, w$ we get $d \mid a^{2}$ which implies $d \mid a$ as $d$ is square-free. Write $a=d a^{\prime}$ so that $d\left(a^{\prime}\right)^{2}-\frac{w}{d} b^{2}-\frac{z}{d} c^{2}=0$ shows that (iii) holds. Finally, if (iii) holds with $(a, b, c)$, multiply $d a^{2}-\frac{w}{d} b^{2}-\frac{z}{d} c^{2}=0$ with $d$ to see that (ii) holds.

Now, by Legendre's theorem on ternary integral quadratic forms [5. Proposition 5.11] item (iii) above is equivalent to the conditions in the statement of the proposition.

Remark. Proposition 2.2 allows us to present explicitly the set $Z \backslash n_{\ell / \mathbb{Q}}\left(\ell^{*}\right)$ which exhausts $\left(\mathbb{Q}^{*} / n_{\ell / \mathbb{Q}}\left(\ell^{*}\right)\right)^{\circ}$. However, in order to get a transversal as in Proposition 1.1 (iii) we need an exhausting set which also is irredundant.

Take $w, w^{\prime} \in Z \backslash n_{\ell / \mathbb{Q}}\left(\ell^{*}\right)$. Then, $w, w^{\prime}$ represent the same coset in $\left(\mathbb{Q}^{*} / n_{\ell / \mathbb{Q}}\left(\ell^{*}\right)\right)^{\circ}$ and only if $\frac{w}{w^{\prime}} \in n_{\ell / \mathbb{Q}}\left(\ell^{*}\right)$ which holds if and only if $\frac{\left(w^{\prime}\right)^{2}}{d^{2}} \cdot \frac{w}{w^{\prime}}=\frac{w^{\prime} w}{d^{2}} \in n_{\ell / \mathbb{Q}}\left(\ell^{*}\right)$. Now, $\frac{w^{\prime} w}{d^{2}} \in Z$ and we can use Proposition 2.2 to answer the question whether $\frac{w^{\prime} w}{d^{2}} \in$ $n_{\ell / \mathbb{Q}}\left(\ell^{*}\right)$. Thus, we can use Proposition 2.2 in order to successively discard elements of $Z \backslash n_{\ell / \mathbb{Q}}\left(\ell^{*}\right)$ that prevent irredundance, hence we have an "infinite method" of reducing $Z \backslash n_{\ell / \mathbb{Q}}\left(\ell^{*}\right)$ to a transversal of $\left(\mathbb{Q}^{*} / n_{\ell / \mathbb{Q}}\left(\ell^{*}\right)\right)^{\circ}$. However, more work is required in order to present these transversals explicitly.

We now consider a special case, in which we can explicitly present a transversal of $\left(\mathbb{Q}^{*} / n_{\ell / \mathbb{Q}}\left(\ell^{*}\right)\right)^{\circ}$.

Proposition 2.3. Let $\ell=\mathbb{Q}(i)$ and define $T(\ell)=\left\{z \in Z \mid m_{p}(z)>0\right.$ implies $p \in$ $\left.\mathbb{P}_{3}\right\}$. Then $T(\ell)$ is a transversal of $\left(\mathbb{Q}^{*} / n_{\ell / \mathbb{Q}}\left(\ell^{*}\right)\right)^{\circ}$. 
Proof. In the present case, $n_{\ell / \mathbb{Q}}\left(\ell^{*}\right)=Q_{2}^{*}$. Let $\pi: \mathbb{Q}^{*} \longrightarrow \mathbb{Q}^{*} / Q_{2}^{*}$ denote the quotient map. The following characterization of elements of $Q_{2}$ is stated as an exercise in 6 , p. 352]: $\frac{a}{b} \in Q_{2}$ if and only if $a b \in N_{2}$. This characterization is a straightforward consequence of Fermat's characterization of elements of $N_{2}$ : Given $n \in \mathbb{Z}_{>0}$ we have $n \in N_{2}$ if and only if $m_{p}(n)$ is even for all $p \in \mathbb{P}_{3}$ (cf. [5, Theorem 2.15]).

Take $q \in \mathbb{Q}^{*}$ such that $\pi(q)$ is not the identity in $\mathbb{Q}^{*} / Q_{2}^{*}$. Since $\mathbb{Q}_{\mathrm{sq}}^{*} \subset Q_{2}^{*}$ there is $z \in Z$ such that $\pi(q)=\pi(z)$ and since $p \in N_{2} \subset Q_{2}$ for each $p \in \mathbb{P}_{1}$ can even take $z \in T(\ell)$. Thus $T(\ell)$ exhausts $\mathbb{Q}^{*} / Q_{2}^{*}$.

Take now $z, z^{\prime} \in T(\ell)$ and suppose $\pi(z)=\pi\left(z^{\prime}\right)$. Then, $\frac{z}{z^{\prime}} \in Q_{2}^{*}$, so $z z^{\prime} \in N_{2}$. Since $z, z^{\prime}$ are square-free integers only divisible by primes in $\mathbb{P}_{3}$ we must have $z=z^{\prime}$ and $T(\ell)$ is a transversal of $\mathbb{Q}^{*} / Q_{2}^{*}$ as desired.

Remark. By 4 , Corollary 4.6] $\mathscr{S}_{\ell}(\mathbb{Q})$ coincides with the category of all four-dimensional Hurwitz division algebras over $\mathbb{Q}$ that contain $\ell$ as a subfield and $\mathbb{Q} V$-submodule. Four dimensional Hurwitz algebras are also known as four-dimensional unital composition algebras or quaternion algebras. Proposition 2.3 yields a classification of the four-dimensional rational Hurwitz algebras that admit $\mathbb{Q}(i)$ as a subfield and $\mathbb{Q} V$-submodule.

\subsection{On $\mathscr{N}(\mathbb{Q})$}

In this section we produce families of algebras in $\mathscr{N}_{\ell}(\mathbb{Q})$ for each $\ell \in \mathscr{L}$ by producing families of admissible triples to which the construction from Section 1 is applied. Considering the definition of $C_{\ell}$, equations (11), (2), and $\ell=\mathbb{Q}(\sqrt{z})$, we have for general $c \in \mathbb{Q}^{3}$ that $c \in C_{\ell}$ if and only if the system

$$
\left\{\begin{array}{l}
0=x_{1}^{2}+z\left(1-2 c_{1}\right) x_{2}^{2}-\left(c_{2}+c_{3}\right) y_{1}^{2}+z\left(c_{3}-c_{2}\right) y_{2}^{2} \\
\left(1-c_{1}\right) x_{1} x_{2}=c_{2} y_{1} y_{2}
\end{array}\right.
$$

admits the trivial solution $\left(x_{1}, x_{2}, y_{1}, y_{2}\right) \in \mathbb{Q}^{4}$ only. Since this system is homogeneous, the existence of a non-trivial rational solution is equivalent to the existence of a primitive integral solution.

In the following proposition we produce three-parameter families of triples in $C_{\ell}$ by choosing $c$ so that the right hand side of the first equation in (44) is always non-negative.

Proposition 2.4. Take $z \in Z$ and set $\ell=\mathbb{Q}(\sqrt{z})$.

(i) If $z>0$ then $P(\ell):=\left\{\left(c_{1}, c_{2}, c_{3}\right) \mid c_{1}<\frac{1}{2} \wedge c_{2}<0 \wedge c_{2}<c_{3}<-c_{2}\right\} \subset C_{\ell}$.

(ii) If $z<0$ then $P(\ell):=\left\{\left(c_{1}, c_{2}, c_{3}\right) \mid c_{1}>\frac{1}{2} \wedge c_{3}<0 \wedge c_{3}<c_{2}<-c_{3}\right\} \subset C_{\ell}$.

Proof. For (i), $c_{1}<\frac{1}{2}$ ensures that $z\left(1-2 c_{1}\right)>0$. From $c_{3}>c_{2}$ we get $z\left(c_{3}-c_{2}\right)>0$ and from $c_{3}<-c_{2}$ we get $c_{2}+c_{3}<0$ and hence $-\left(c_{2}+c_{3}\right)>0$ so the right hand side of the first equation of (4) is non-negative for each $\left(x_{1}, x_{2}, y_{1}, y_{2}\right) \in \mathbb{Q}^{4}$ and hence (4) only admits the trivial solution, implying $\left(c_{1}, c_{2}, c_{3}\right) \in C_{\ell}$. Similar verifications yield (ii). 
In the following corollary we extract from each three-parameter family $P(\ell)$ of the previous proposition a two-parameter irredundant subfamily $\tilde{P}(\ell) \subset P(\ell)$.

Corollary 2.5. Take $z \in Z$, set $\ell=\mathbb{Q}(\sqrt{z})$ and let $S(\ell) \subset \mathbb{Q}^{*}$ be as in Proposition 2.1, $P(\ell)$ as in Proposition 2.4.

(i) If $z>0$ then $\tilde{P}(\ell):=\left\{\left(r, s, \frac{1}{2}\right) \mid r<\frac{1}{2} \wedge s \in S(\ell)_{<0}\right\} \subset C_{\ell}$ is an irredundant subset of $P(\ell)$.

(ii) If $z<0$ then $\tilde{P}(\ell):=\left\{(r, s, s-1) \mid r>\frac{1}{2} \wedge s \in S(\ell)_{<0}\right\} \subset C_{\ell}$ is an irredundant subset of $P(\ell)$.

Proof. This corollary is straightforward consequence of Proposition 1.1 (i), the definition of $P(\ell)$ and the fact that $S(\ell)$ is a transversal of $\left(\mathbb{Q}^{*} /\left(\mathbb{Q}^{*} \cap \ell_{\mathrm{sq}}\right)\right)^{\circ}$.

Remark. Given $c=\left(c_{1}, c_{2}, c_{3}\right) \in C_{\ell}$ we have from [4, Prop 3.3] that $A(\ell, c) \in \mathscr{K}(\mathbb{Q})$ if and only if $c_{1}=c_{3}=0$ and $A(\ell, c) \in \mathscr{S}(\mathbb{Q})$ if and only if $\left(c_{1}, c_{2}\right)=(1,0)$. Thus, for each $c \in \tilde{P}(\ell)$ as above we have $A(\ell, c) \in \mathscr{N}_{\ell}(\mathbb{Q})$.

We now show how further families of triples in $C_{\ell}$ can be found for particular $\ell$ by choosing $c$ such that various number theoretic obstructions guarantee that the system (4) only admits the trivial solution.

Proposition 2.6. Take $z \in Z$ such that $m_{p}(z)>0$ for some $p \in \mathbb{P}_{3}$, and fix such p. Then, if $c=\left(c_{1}, c_{2}, c_{3}\right) \in \mathbb{Z}^{3}$ satisfies $1-2 c_{1} \equiv 1(\bmod p), c_{2} \equiv-1(\bmod p)$, $c_{3} \equiv 0(\bmod p)$ we have $c \in C_{\ell}$.

Proof. Suppose $c$ is as above and, towards a contradiction, that (4) admits a primitive integral solution $\left(x_{1}, x_{2}, y_{1}, y_{2}\right)$. Considering the first equation of (44) modulo $p$ we get $x_{1}^{2}+y_{2}^{2} \equiv 0(\bmod p)$. Since $p \in \mathbb{P}_{3}$ we get, by [5, Lemma 2.14] that $p \mid x_{1}, x_{2}$. Thus,

$$
p^{2} \mid x_{1}^{2}-\left(c_{2}+c_{3}\right) y_{1}^{2}=z\left(\left(c_{2}-c_{3}\right) y_{2}^{2}-\left(1-2 c_{1}\right) x_{2}^{2}\right) .
$$

Since $z$ is square-free, we get $p \mid\left(c_{2}-c_{3}\right) y_{2}^{2}-\left(1-2 c_{1}\right) x_{2}^{2}$. By assumptions on $c_{1}, c_{2}, c_{3}$ we get $y_{2}^{2}+x_{2}^{2} \equiv 0(\bmod p)$ so $p \mid x_{2}, y_{2}$, contradiction to $\left(x_{1}, x_{2}, y_{1}, y_{2}\right)$ being a primitive solution of (4).

Remark. Note that, for the same reason as stated in the remark after Corollary 2.5, for all triples $c$ produced in Proposition 2.6 we have $A(\ell, c) \in \mathscr{N}_{\ell}(\mathbb{Q})$.

In the remaining part of this section we investigate the case $\ell=\mathbb{Q}(i)$ further.

Proposition 2.7. $\quad$ (i) The one-parameter family $P_{1}(\mathbb{Q}(i)):=\left\{\left(\frac{1-q}{2}, 0,-1\right) \mid q \in \mathbb{Q}_{>0} \backslash Q_{2}\right\}$ satisfies $P_{1}(\mathbb{Q}(i)) \subset C_{\mathbb{Q}(i)}$ and is irredundant.

(ii) The one-parameter family

$P_{2}(\mathbb{Q}(i)):=\left\{(1, n, 0) \mid n \in Z_{<0}\right.$ such that there exists $p \in \mathbb{P}_{3}$ with $\left.m_{p}(n)=1\right\}$ satisfies $P_{2}(\mathbb{Q}(i)) \subset C_{\mathbb{Q}(i)}$ and is irredundant. 
Proof. (i): Let $q \in \mathbb{Q}_{>0} \backslash Q_{2}$ and set $\left(c_{1}, c_{2}, c_{3}\right)=\left(\frac{1-q}{2}, 0,-1\right)$. Then the system (44) looks as follows

$$
\left\{\begin{array}{l}
0=x_{1}^{2}-q x_{2}^{2}+y_{1}^{2}+y_{2}^{2} \\
\left(\frac{q+1}{2}\right) x_{1} x_{2}=0
\end{array}\right.
$$

and the second equation implies that a solution $\left(x_{1}, x_{2}, y_{1}, y_{2}\right) \in \mathbb{Q}^{4}$ must satisfy $x_{1}=0$ or $x_{2}=0$.

If $x_{2}=0$ then the first equation in (5) is $0=x_{1}^{2}+y_{1}^{2}+y_{2}^{2}$ which implies $x_{1}=y_{1}=y_{2}=0$. If $x_{1}=0$ then the first equation becomes $q x_{2}^{2}=y_{1}^{2}+y_{2}^{2}$, which implies $q \in Q_{2}$ unless $x_{2}=0$, which was seen to imply that $y_{1}=y_{2}=0$. Thus, the system (5) only admits the trivial solution and we conclude that $P_{1}(\mathbb{Q}(i)) \subset C_{\mathbb{Q}(i)}$.

For irredundance, we merely note that the elements of $P_{1}(\mathbb{Q}(i))$ all have different first components whence the claim follows immediately from Proposition 1.1 (i).

(ii): For $\left(c_{1}, c_{2}, c_{3}\right)=(1, n, 0)$ the system (4) becomes

$$
\left\{\begin{array}{l}
0=x_{1}^{2}+x_{2}^{2}-n y_{1}^{2}+n y_{2}^{2} \\
0=n y_{1} y_{2}
\end{array}\right.
$$

and we assume that (6) admits a primitive integral solution $\left(x_{1}, x_{2}, y_{1}, y_{2}\right)$. Then, by the second equation, we have $y_{1}=0$ or $y_{2}=0$. If $y_{2}=0$ then the first equation implies $x_{1}=x_{2}=y_{1}=0$. If $y_{1}=0$, then the first equation implies $-n y_{2}^{2}=x_{1}^{2}+x_{2}^{2}$. Fix $p \in \mathbb{P}_{3}$ such that $m_{p}(n)=1$. Then, $p \mid x_{1}^{2}+x_{2}^{2}$ and hence $m_{p}\left(x_{1}^{2}+x_{2}^{2}\right)$ is even. By assumption of $\left(x_{1}, x_{2}, y_{1}, y_{2}\right)$ being primitive, we have $p \nmid y_{2}$. Thus, $m_{p}\left(-n y_{2}^{2}\right)$ is equal to 1 while $m_{p}\left(x_{1}^{2}+x_{2}^{2}\right)$ is even, contradiction, and we conclude that $P_{2}(\mathbb{Q}(i)) \subset$ $C_{\mathbb{Q}(i)}$.

For irredundance, we note that the middle component runs through a subset of the transversal $S(\mathbb{Q}(i))$ of $\left(\mathbb{Q}^{*} /\left(\mathbb{Q}_{\mathrm{sq}}^{*} \cap \mathbb{Q}^{*}\right)\right)^{\circ}$ given in Proposition 2.1.

Considering the first components of elements in $\tilde{P}(\mathbb{Q}(i)), P_{1}(\mathbb{Q}(i))$ and $P_{2}(\mathbb{Q}(i))$ (the families obtained in Corollary 2.5 and Proposition 2.7) we conclude with Proposition 1.1 that $\tilde{P}(\mathbb{Q}(i)) \cup P_{1}(\mathbb{Q}(i))$ and $P_{1}(\mathbb{Q}(i)) \cup P_{2}(\mathbb{Q}(i))$ are irredundant subsets of $C_{\mathbb{Q}(i)}$. Since the third component of a given element in $P_{2}(\mathbb{Q}(i))$ is 0 and the third component of a given element in $\tilde{P}(\mathbb{Q}(i))$ is non-zero we conclude with Proposition 1.1 that $\tilde{P}(\mathbb{Q}(i)) \cup P_{2}(\mathbb{Q}(i))$ is irredundant and hence the family defined by $F(\mathbb{Q}(i))=\tilde{P}(\mathbb{Q}(i)) \cup P_{1}(\mathbb{Q}(i)) \cup P_{2}(\mathbb{Q}(i))$ is an irredundant four-parameter family of admissible triples in $C_{\mathbb{Q}(i)}$. Furthermore, $A(\mathbb{Q}(i), c) \in \mathscr{N}_{\mathbb{Q}(i)}(\mathbb{Q})$ for each $c \in F(\mathbb{Q}(i))$.

In this section, we studied $\mathscr{N}(\mathbb{Q})$ locally, i.e. via the components of its covering by local subcategories in (3) indexed by $\mathscr{L}$. For each field $\ell \in \mathscr{L}$ we studied the system (4) with the production of admissible triples $c \in C_{\ell}$ such that $A(\ell, c) \in$ $\mathscr{N}(\ell / \mathbb{Q})$ in mind. The nature of $\mathbb{Q}$ gives rise to two complexities: since $|\mathscr{L}|=\infty$ there is an abundance of local subcategories to consider and since $\mathbb{Q}$ is far from being algebraically closed, there should be many possibilites of $c$ such that (4) only admits the trivial solution. By varied approaches from elementary number theory we produced parametrized families of triples $c \in C_{\ell}$ such that $A(\ell, c) \in \mathscr{N}_{\ell}(\mathbb{Q})$ for each $\ell$, establishing richness of $\mathscr{N}_{\ell}(\mathbb{Q})$, but a classification of $\mathscr{N}_{\ell}(\mathbb{Q})$ seems distant. 


\section{References}

[1] E. DARPÖ AND A. RochDI, Classification of the four-dimensional powercommutative real division algebras, Proceedings of the Royal Society of Edinburgh Section A: Mathematics, 141 (2011), pp. 1207-1223.

[2] E. Dieterich, Zur Klassifikation vierdimensionaler reeller Divisionsalgebren, Mathematische Nachrichten, 194 (1998), pp. 13-22.

[3] _ Classification, automorphism groups and categorical structure of the twodimensional real division algebras, Journal of Algebra and its applications, 4 (2005), pp. 517-538.

[4] _- On four-dimensional division algebras over fields of characteristic not 2, Submitted for publication, (2017).

[5] I. Niven, H. S. Zuckerman, and H. L. Montgomery, An introduction to the theory of numbers, John Wiley \& Sons, 1991.

[6] W. Sierpiński, Elementary theory of numbers, Państwowe Wydawnictwo Waukowe, Warszawa, 1964. 